\title{
Land Cover Recognition using Min-Cut/Max-Flow Segmentation and Orthoimages
}

\author{
Sergejs Kodors \\ Rezekne Higher Education Institution, \\ Atbrivoshanas Str. 115, Rezekne, LV-4601
}

\begin{abstract}
The geospatial information is significant for many socio-technical activities like urban planning, the prediction of natural hazards, the monitoring of land use, weather forecasting, cadastral surveys etc. It is possible to acquire geospatial information from a distance using remote sensing technologies, but remotely sensed images don't have semantics without a previous recognition. The classification of geospatial information is expensive and time consuming process. The paper describes the automatic land cover recognition method, which is based on mincut/max-flow segmentation. The raw data are othoimages with a high resolution. The proposed method is tested and evaluated by Cohen's kappa coefficient.
\end{abstract}

Keywords: land cover, min-cut/max-flow segmentation, orthoimage, recognition.

\section{INTRODUCTION}

It is very important to acquire and to analyze the state of an environment before making some decisions. Therefore geospatial information is the significant information, which is used in many sociotechnical activities like an urban planning, the prediction of natural hazards, the monitoring of land use, weather forecasting, cadastral surveys etc.

Nowadays it is possible to acquire the geospatial information from a distance using the modern technologies like aerial vehicles, satellites or spacecrafts. The remote sensing is the scientific field how to acquire the geospatial information without a physical contact. All remote sensing techniques are based on the processes of energy emission and reflection from the observed objects of a land surface. These techniques are particularly effective, if a monitoring region is very large and problematically accessed for the ground surveys.

If the geospatial information can be acquired with a single attempt, the interpretation of this information is an expensive and time consuming process.

The paper describes the automatic land cover recognition method, which is based on min-cut/maxflow segmentation. The land cover recognition is the applied science of the artificial intellect. The goal of the land cover recognition is the classification of the geospatial objects using the remote sensing data. The proposed method uses orthoimages as the raw data.

Min-cut/max-flow method separates images into segments using the prior knowledge about the locations of an object and a background. Traditionally the locations of the object and the background, which are called seeds, are manually marked, while the unmarked pixels are labeled in the process of the segmentation. If the manual part is replaced by the automatic method, when the object and the background have the semantic meaning (segments are classes), then min-cut/max-flow segmentation can be used as the recognition method.

The proposed method of the land cover recognition is based on the logical reasoning, which is defined using the color histograms of orthoimages. The logical reasoning is the simplest approach, which can be replaced by the more complicated supervised or unsupervised methods. As a result, the paper provides the framework for the plenty different realizations of the recognition systems, which can be applied for different purposes.

\section{ORTHOIMAGE AND GEOSPATIAL INFORMATION}

An orthoimage is the digital type of an orthophotograph. The orthphotograph is the aerial panorama of the land surface, which is orthorectified (the aerial photograph, which has not distortions caused by a perspective). Traditionally the orthophotograph was produced using orthophotoscope instruments. The usage of computer software systems instead of the hardware to create the digital orthophotograph was initiated in the late 1970s [1]. The orthoimage is produced by the rectification 
algorithms, which analyze the visibility of the geospatial objects using the perspective images and photo orientation parameters [1], [2].

The geographical information systems (GIS) use two representation formats of the geospatial information, which are called raster and vector data formats. The orthoimages belong to the group of the raster format. The raster images are represented as a regular grid, where the pixel is the smallest element. The raster images are widely used in the computer graphics, but the geospatial raster images have two additional features: a geographical location and the proportional geometrical sizes in the real world. The smaller spatial size of the pixel identifies the higher resolution of the image. The medium resolution of the orthoimage is $10-100$ meters per one pixel [3].

The pixel-based methods of the land cover recognition are less effective than the object-based methods, when the image resolution increases [3], [4]. The pixel-based methods classify each pixel independently, but the object-based methods classify the whole segments. The segments are the sets of the pixels, which have the similar features like a color, a texture and brightness. The modern orthoimages can have the resolution, which is equal to the half of the centimeter (2012) [5]; therefore the segmentation is the incredibly important process to recognize the modern orthoimages. As a result, the land cover recognition method, which is based on min-cut/maxflow segmentation, gives strong promises to classify the land cover.

\section{IMAGE SEGMENTATION}

The image segmentation is the process of the labeling each pixel, where the label is the unique number, which identifies the similar regions.

The recognition is the classification, which labels each pixel too, but the label has the semantic meaning and identifies the category; therefore the segmentation methods can be adopted for the classification problems.

The different methods of the image segmentation were proposed by different authors:

- the edge detection [6-8];

- the watershed algorithm [9];

- the expectation-maximization method [7];

- the genetic algorithms [7], [10];

- Kohenen's self-organizing maps [10];

- min-cut/max-flow segmentation [11]-[14].

The paper describes the land cover recognition method, which is based on min-cut/max-flow segmentation.

\section{MIN-CUT/MAX-FLOW SEGMENTATION}

Min-cut/max-flow segmentation is based on the next principles:
- The adjacent pixels are connected by some energy, which must be applied to break these connections.

- The pixels of the same segment have the higher energy potential, then the pixels between two segments.

- The goal of the method is to find a solution, when the minimal energy is applied to break the image into two parts: an object and a background.

- The image is transformed into the weighted directed mathematical graph to solve the energy minimization problem.

- The energy minimization problem is solved using the algorithms of the maximal flow.

So, min-cut/max-flow segmentation is organized as an energy minimization problem, when the solution is found as the maximal flow through a network, which is constructed as the transformation of image into the mathematical graph, which describes the connections among the pixels and pixels' similarities.

\section{A. Mathematical Graph}

The mathematical graph is the collection of two dependant sets: $G=\{V ; E\}$, where $V$ is the vertices, but $E$ - the edges. The vertices are the nodes or the elements, which are connected by the edges. The edges of the graph are written as $(a, b) \in E$, where $\{a$, $b\} \in V$. If a movement between two nodes is restricted by one direction, the graph is called directed. If the edge has the weight, which represents the cost of the movement, the graph is called weighted. The visual representation of the mathematical graph is depicted in Fig. 1.

The image transformation is executed using the next principles:

- Each pixel is replaced by the node of the graph (see Fig. 2).

- The nodes of the adjacent pixels are connected by two edges: the edge from $p$ to $q$ and the reverse edge from $q$ to $p$ (see Fig. 2). The edges between two nodes of the adjacent pixels are called $N$-links [13].

- The weights of the $N$-links are calculated by the function of smooth cost $V_{p, q}\left(L_{p}, L_{q}\right)$, where $L$ is the labels of adjacent vertices $p$ and $q$.

- 4-path or 8-path schema can be used to define the adjacent pixels.

- Two terminal nodes are added to the graph: one is called source $S$, but another - sink $T$ (see Fig. 2).

- The source $S$ is connected with each node of the pixel by the edge, and each node of the pixel is connected with the sink $T$ by the edge (see Fig. 2). The edges, which connect the nodes of the pixels with the terminals, are called $T$-links [13]. 
- The weights of $T$-links are calculated by the function of label cost $D_{p}\left(L_{p}\right)$.

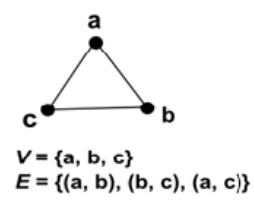

a)

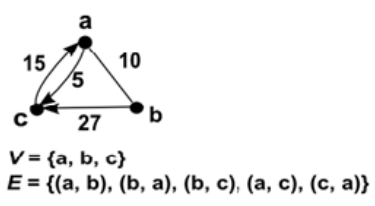

b)
Fig. 1. a) Undirected mathematical graph, b) weighted directed mathematical graph

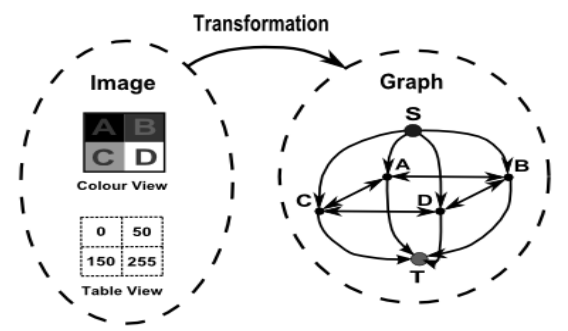

Fig. 2. Principal schema of image transformation into mathematical graph

- The terminal $S$ is associated with the segment ' 0 ' and the terminal $T$ - with the segment ' 1 '.

The weights of the mathematical graph must satisfy the next conditions:

- The weights must be assigned to each edge of the graph [11], [13].

- The weights can be calculated using any nonnegative symmetric function [11].

- The weights between the adjacent pixels must be higher, if the pixels belong to the same segment, and they must be lower in the border of two segments [11], [12], and [14].

- The weight of the edge $(p, q)$ may differ from the weight of the reverse edge $(q, p)$ [13].

\section{B. Energy minimization}

When the image transformation into the mathematical graph is completed, the algorithm starts to search the optimal solution. The optimal solution is the minimal cut, which divides the image into two parts using the minimal energy. The volume of the energy, which must be applied to separate the image, is calculated using the formula (1):

$$
E(L)=\sum D_{p}\left(L_{p}\right)+\sum V_{p, q}\left(L_{p,} L_{q}\right),
$$

where $p$ - a current pixel, $q$ - an adjacent pixel, $L-$ the set of the labels, which indicate the segments of the pixels, $D_{p}\left(L_{p}\right)$ - the cost to assign the label $L_{p}$ to the pixel $p, V_{p, q}\left(L_{p}, L_{q}\right)$ - the energy, which must be applied to break the connection between the pixels $p$ and $q$ [11], [13]. The component $D_{p}\left(L_{p}\right)$ is called label cost, but $V_{p, q}\left(L_{\mathrm{p}}, L_{q}\right)$ is called smooth cost [15]. The label cost and the smooth cost are used to calculate the weights of the mathematical graph.

There are the examples of minimal cuts in Fig. 3:

- If the cut is $C=\{(S, A),(S, B)\}$, then the nodes $A$ and $B$ remain connected with the sink $T$, that means they belong to segment ' 1 ' and the minimal energy is $D_{A}(0)+D_{B}(0)$ (see Fig. 3a).

- If the cut is $C=\{(A, T),(B, T)\}$, then the nodes $A$ and $B$ remain connected with the source $S$, that means they belong to segment ' $O$ ' and the minimal energy is $D_{A}(1)+D_{B}(1)$ (see Fig. 3b).

- If the cut is $C=\{(S, A),(A, B),(B, T)\}$, then the node $A$ remains connected with the sink $T$, but $B$ - with the source $S$ and the minimal energy is $D_{A}(0)+D_{B}(1)+V_{A, B}(0,1)$ (see Fig. 3c).

\section{Maximal flow}

According to the theorem of Ford-Fulkerson, the problem of the minimal cut can be solved by finding the maximal flow, when the edges are associated with the pipelines of water, but the weights of the graph are presented as a flow capacity [11], [13]. According to the principle, that "the pixels of the same segment have the higher potential energy, then the pixels of the border", the boundary pixels create the bottlenecks for the flow from the source $S$ to the sink $T$ and the bottlenecks are the min-cut of the graph (see Fig. 4), which can be find by the logical expression (2):

Edge is boundary, iff flow capacity $=$ flow $A N D$ flow $>0$.

There is the example of the bottleneck in Fig. 4:

- $(A, B)$ : flow capacity $>$ flow, $(A, B)$ is not the boundary.

- $(B, C)$ : flow capacity $=$ flow, $(B, C)$ is the boundary. $B$ belongs to the source, but $C$ - to the sink, according to the direction of the flow (flow $>0)$.

- $(C, D)$ : flow capacity $>$ flow, $(C, D)$ is not the boundary.

The maximal flow can be found, for example, by Dinic's algorithm, which is based on two principles:

- The shortest paths from $S$ to $T$ must be filled firstly.

- A residual graph is constructed in all iterations. The residual graph represents the remaining paths and their flow capacity. The flow capacity of $T$-links is calculated by the formula $f(t)=f(t$ 1) $-d x(t)$, where $f$ - new flow capacity, $t-\mathrm{a}$ current iteration and $d x$ - a launched flow in the iteration $t$. When the flow capacity of $N$-link is decreased, the flow capacity of the reverse edge must be increased by the formula $f(t)=f(t-1)+$ $d x(t)$.

\section{Seeds}

The paper describes the seeds-based method. The seeds are the marked pixels, which identify the location of the object and the background. Seeds 
organize the input and output points of the water flow. These seeds are converted by the label cost function $D_{p}\left(L_{p}\right)$ : if pixel is marked, $T$-link has the flow capacity equal to infinity, else it is 0 .

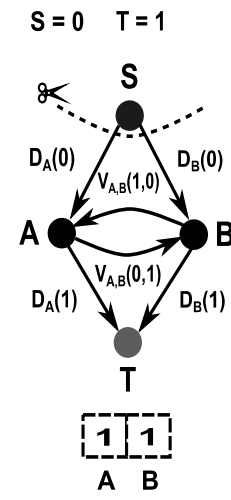

a)

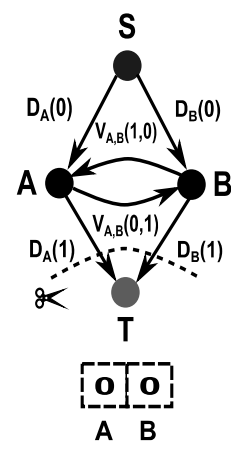

b)

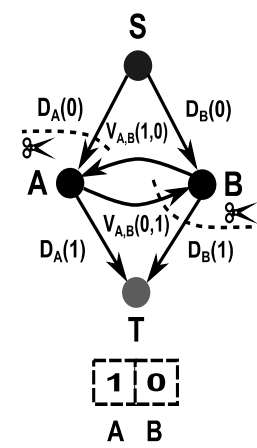

c)
Fig. 3. Different cases of minimal cut

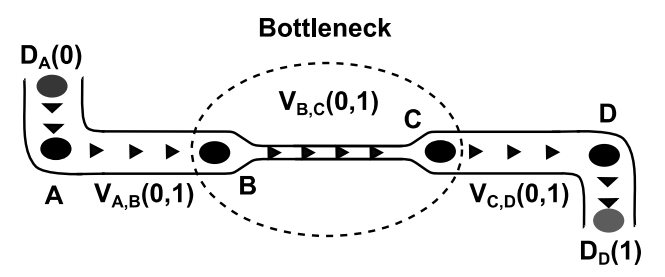

Fig. 4. Bottleneck of water flow

\section{E. Algorithm of Min-Cut/Max-Flow Segmentation}

1) select the prior knowledge about the image;

2) define the label cost $D_{p}\left(L_{p}\right)$;

3) define the smooth cost $V_{p, q}\left(L_{p}, L_{q}\right)$;

4) transform image into the weighted directed mathematical graph;

5) find the maximal flow;

6) mark the pixels of the source and the sink;

7) mark the pixels with the bottlenecks using the function (2);

8) fill in the segments using the pixels of the source and the sink as start points;

9) mark the pixels of the border as the category of the source.

\section{$\mathrm{V}$ LAND COVER RECOGNITION USING MIN-CUT/MAX-FLOW SEGMENTATION}

The paper discusses the land cover recognition, which classifies the pixels into three categories: water, field and forest.

The orthoimages with the high resolution have too many classes, which can be sensed: cars, road markings, parasols and other small objects. All these small objects create a noise for the land cover recognition. This noise can be filtered by the small resolution, therefore the resolution of $2.5 \mathrm{~m}$./pix. is selected.

The land cover recognition using min-cut/max-flow segmentation is based on the principle, that the seeds of the object and the background are placed automatically using some recognition method, therefore it is the recognition system, which integrates two modules:

- A seeds marker, which marks the position of the objects using some recognition method.

- A segmenter, which segments the objects using the seeds defined by seeds maker.

As a result, the quality of the recognition depends on the potential of the min-cut/max-flow segmentation, which itself depends on the quality of the seeds maker.

The quality of the land cover recognition is assessed by Cohen's kappa coefficient (3) [16], [17]:

$$
k=\frac{N \sum_{i=1}^{r} x_{i i}-\sum_{i=1}^{r}\left(x_{i+} \cdot x_{+i}\right)}{N^{2}-\sum_{i=1}^{r}\left(x_{i+} \cdot x_{+i}\right)},
$$

where $r$ indicates the number of rows in the error matrix (the headers of columns and rows are categories, the rows indicates the result of classification, but the columns - the waiting result), $x_{i i}$ - the number of observations in the row $i$ and the column $i, x_{i+}$ - the sum of the $i$-th row observations, $x_{+i}$ - the sum of the $i$-th column observations, $N$ - the number of the elements.

Cohen's kappa evaluation method needs the classified image and the sample of waiting result, which are compared. The orthoimage is depicted in Fig. 5a. and the waiting result of the classification is depicted in Fig. 5b:

\section{A. Potential of min-cut/max-flow segmentation}

If the seeds are manually marked by a human, then it is possible to accept, that the seeds maker has the best possible quality. As a result, it is possible to evaluate the potential of min-cut/max-flow method as the method of the land cover recognition, because the result only depends on the quality of the segmenter in this case. The experiment has showed the result of Cohen's kappa coefficient equal to 0.93 (see Fig. 5c).

\section{B. Automatization using logical reasoning}

According to the chapter $4 \mathrm{E}$, the first step is to select the prior knowledge about the image. It is done using the color histograms of three categories (see Fig. 6-8). It is possible to see that the categories have the intersections, therefore the disjoint ranges are selected for the logical reasoning of the seeds maker, but the intersections will be classified by the segmenter. But the min-cut/max-flow algorithm divides the image into two segments, therefore the land cover recognition must be organized as two steps process:

1) water/land segmentation: $D_{p}$ ( 'water' ) $=\infty$, if $x_{p}<28$; and $D_{p}$ ( 'surface' ) $=\infty$, if $x_{p}>$ 39; 
2) forests/fields segmentation: $D_{p}$ ( 'forest' ) = $\infty$, if $x_{p}<79$; and $D_{p}$ ( 'field' ) $=\infty$, if $x_{p}>$ 111.

The smooth cost can be defined using the function (4) and Manhattan distance (5):

$$
V_{p, q}\left(L_{p}, L_{q}\right)= \begin{cases}\infty, & \text { if }\left|x_{p}-x_{q}\right|=0 \\ \frac{1}{\left|x_{p}-x_{q}\right|}, & \text { if }\left|x_{p}-x_{q}\right|>0\end{cases}
$$

$\Delta x=\mid x_{p}$, red $-x_{q} \cdot$ red $|+| x_{p}$.green $-x_{q} \cdot$ green $|+| x_{p}$, blue $-x_{q} \cdot$ blue $\mid$.

The function (4) satisfies all conditions described in the $4 \mathrm{~A}$ chapter.

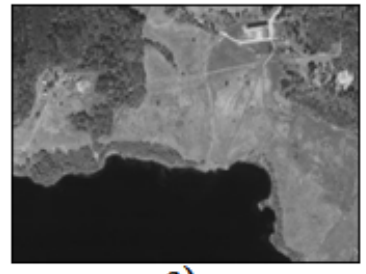

a)

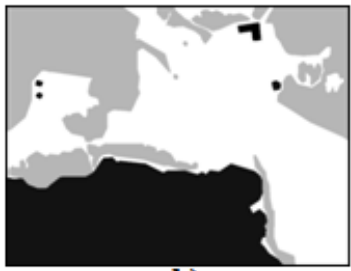

b)
The method was evaluated by Cohen's kappa coefficient using the image of Fig.5a. The results of the experiment are depicted in Fig.5d and in Table 1.

TABLE I.

ASSESSMENT OF LAND COVER RECOGNITION

\begin{tabular}{|l|l|l|}
\hline & \multicolumn{2}{|l|}{ Land cover recognition } \\
\hline $\begin{array}{l}\text { Cohen's } \\
\text { kappa }\end{array}$ & 0.7458 \\
\hline & User's accuracy & Producer's accuracy \\
\hline Water & $86.27 \%$ & $98.78 \%$ \\
\hline Forests & $94.72 \%$ & $76.04 \%$ \\
\hline Fields & $68.04 \%$ & $79.02 \%$ \\
\hline
\end{tabular}

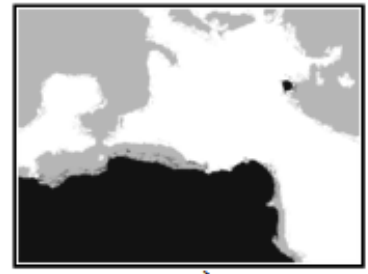

c)

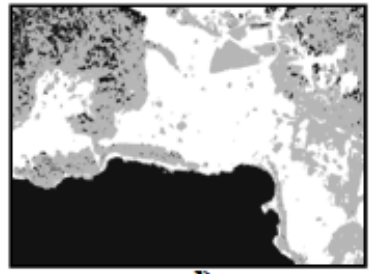

d)

Fig. 5. a) orthoimage; b) waiting result; c) result of segmenter examination; d) result of land cover recognition

\section{Colour Histogram of Water}

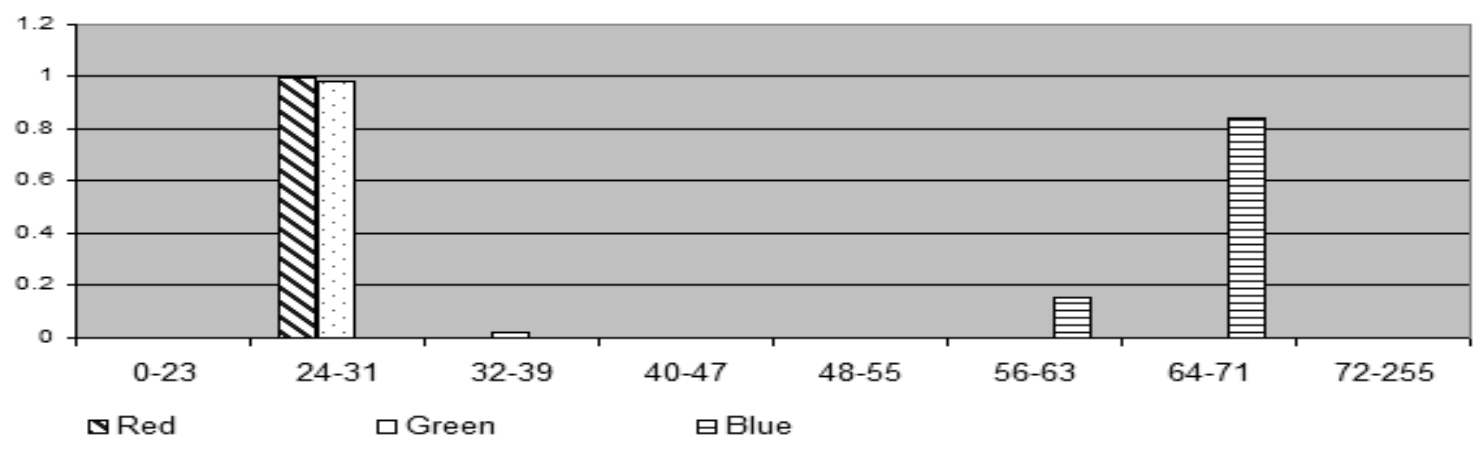

Fig. 6. Colour histogram of water

Colour Histogram of Wood

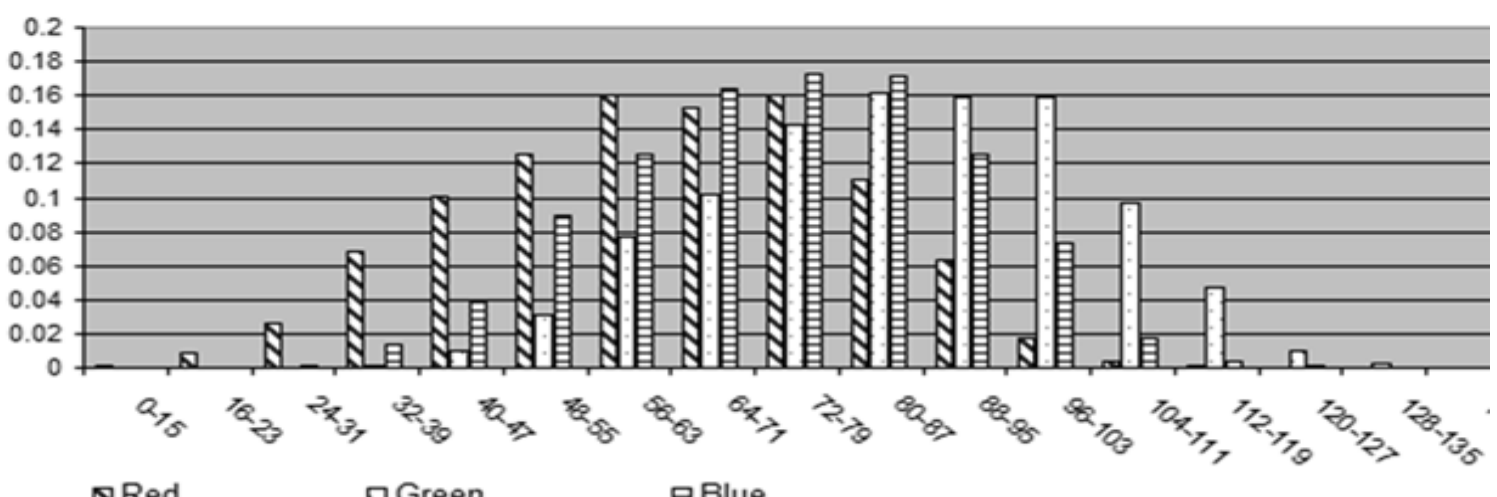

Fig. 7. Colour histogram of forest 


\section{Colour Histogram of Field}

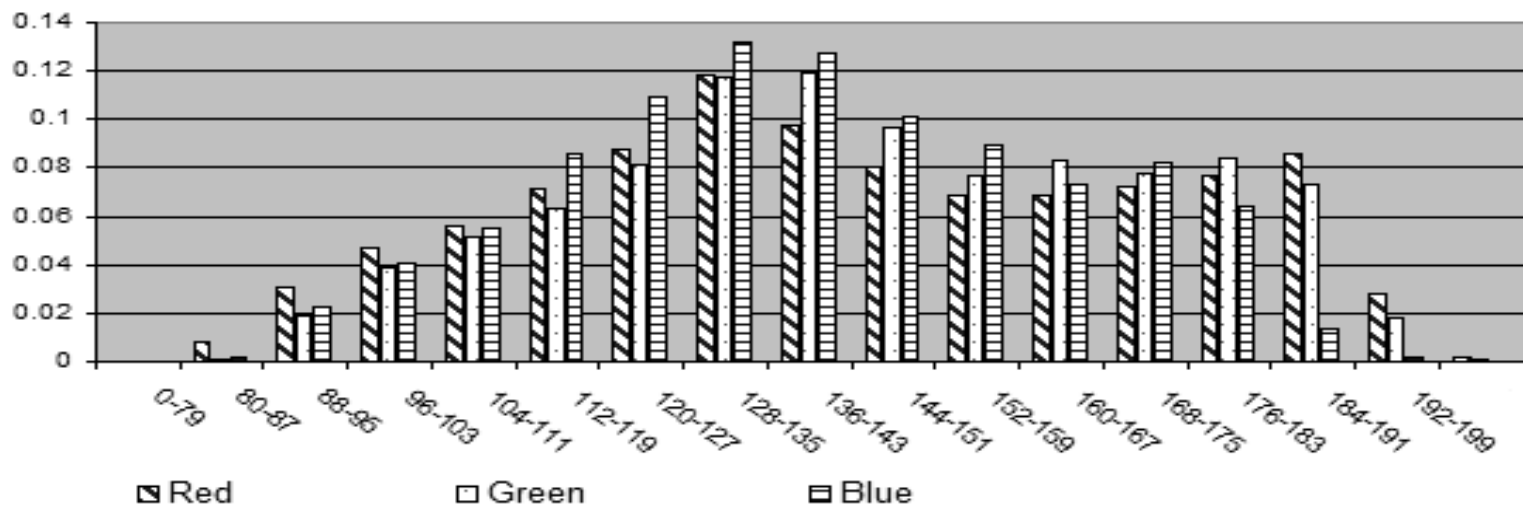

Fig. 8. Colour histogram of field

\section{CONCLUSIONS}

It was proved that the min-cut/max-flow segmentation can be used for the automatic land cover recognition. The method is organized as two step process: the seeds finding and the segmentation; when min-cut/max-flow algorithm classifies the remaining parts using the energy minimization principle.

The experiment was organized in two steps:

- the assessment of the potential of the proposed method using the conditions of the ideal seeds maker (manually marked seeds);

- the assessment of the method using the seeds maker, which was based on the logical reasoning (fully-automatic recognition).

The results of the recognition were measured by Cohen's kappa coefficient, which uses the image of the waiting result to express the quality of recognition.

Changing the seeds maker's algorithm, it is possible to construct the different recognition systems, which have the different quality of the recognition and which can be applied for different computer vision problems. The paper is connected with the land cover recognition problem. The seeds maker of the described solution was based on the logical reasoning, which had been defined using the color histograms of three categories: water, fields and forests. The assessment of this method has showed the result Cohen's kappa coefficient equal to 0.746 .

According to the fact, that the recognition system consists from two parts, its quality depends on each of them. The experiment has showed that min-cut/maxflow segmentation has the potential, which is equal to Cohen's kappa coefficient 0.93 , therefore the seeds maker must be modified to increase the accuracy of the method.

According to the principle, which is used by mincut/max-flow algorithm, the ideal seeds maker must mark the pixels with $100 \%$ precision, when the number of the seeds is not so important, it can be only one point for each simple segment. In this case, the simple segment is the segment, which does not have some subcategories, which can have the greater distances (which can form the smaller bottlenecks) than the distances between the main classes.

\section{ACKNOWLEDGEMENT}

The author expresses gratitude to the State Land Service of Latvia for providing samples for research purposes.

\section{REFERENCES}

[1] Yongwel Sheng, Peng Gong and Gregory S., "Biging.True Orthoimage Production for Forested Areas from Large-Scale Aerial Photographs,” American Society for Photogrammetry and Remote Sensing, Photogrammetric Engineering \& Remote Sensing, Vol. 69, No. 3, pp. 259-266, March 2003.

[2] Fahmi Amhar, Josef Jansa and Christine Ries., “The Generation of True Orthophotos using a 3D Building Model in Conjunction with a Conventional DTM,” IAPRS, Vol. 32, Part 4, pp. 16-22, 1998.

[3] T. Veljanovski, U. Kanjir and K. Ostir., "Object-based image analysis of remote sensing data,” Geodetski Vestnik, vol. 55, no. 4, pp. 678-688, 2011.

[4] Y. Babykalpana and K. ThanushKodi., "Classification of land use land cover change detection using remotely sensed data," International Journal on Computer Science and Engineering (IJCSE), Vol. 3, no. 4, pp. 1638-1644, 2011.

[5] Military Memorial Park, Pákozd, “The world's highest spatial resolution aerial orthophoto map produced by aeroplane," Military Memorial Park, Pákozd. [Online]. Available: www.aerialrecord.com. [Accessed 05.04.2013].

[6] S. S. Al-amri, N. V. Kalyankar and S. D. Khamitkar, "Image Segmentation by using Edge Detection,” International Journal on Computer Science and Engineering, Vol. 2, pp. 804-807, 2010.

[7] Y. Ramadevi, T. Sridevi, B. Poornima and B. Kalyani, "Segmentation and object recognition using edge detection techniques," International Journal of Computer Science \& Information Technology (IJCSIT), Vol. 2, no. 6, pp. 153-161, December 2010.

[8] Sergejs Kodors and Imants Zarembo, Urban Objects Segmentation Using Edge Detection, Environment. Technology. Resources, Proceedings of the 9th International Scientific and Practical Conference. Vol. 2, pp. 26-29, 2013.

[9] G. Ignatov, H. Nikolov, D. Petkov and G. Georgie, "Segmentation of satellite images by means of morphological and object-oriented approaches," International Society of Photogrammetry and Remote Sensing (ISPRS), Vol. 36, part 7, p. 4, May 2006. 
[10] Mohamad Awad, Kacem Chehdi and Ahmad Nasri, "Multicomponent Image Segmentation Using a Genetic Algorithm and Artificial Neural Network,” IEEE Geoscience and Remote Sensing Letters, Vol. 4, No. 4, pp. 571-575, October 2007.

[11] Zhenyu Wu and Richard Leahy, “An Optimal Graph Theoretic Approach to Data Clustering: Theory and Its Application to Image Segmentation,” IEEE Transactions on Pattern Analysis and Machine Intelligence, Vol. 15, No. 11, pp. 1101-1113, November 1993.

[12] Vladimir Kolmogorov and Ramin Zabih, "What Energy Functions Can Be Minimized via Graph Cuts?,” IEEE Transactions on Pattern Analysis and Machine Intelligence, Vol. 26, No. 2, pp. 147-159, February 2004

[13] Yuri Boykov and Vladimir Kolmogorov, “An Experimental Comparison of Min-Cut/Max-Flow Algorithms for Energy Minimization in Vision,” IEEE Transactions on Pattern
Analysis and Machine Intelligence, Vol. 26, No. 9, pp. 11241137, September 2004

[14] Yury Boykov and Gareth Funka-Lea, "Graph Cuts and Efficient N-D Image Segmentation,” International Journal of Computer Vision, Vol. 70, No. 2, pp. 109-131, 2006.

[15] Andrew Delong, Anton Osokin, Hossam N. Isack and Yuri Boykov, "Fast Approximate Energy Minimization with Label Costs,” International Journal of Computer Vision, Vol. 96, no. 1, pp. 1-27, January 2012.

[16] Sergejs Kodors and Imants Zarembo, Land Cover Recognition with Logical Reasoning using Orthophoto Images, RCITD 2013, Proceeding of the 1st Research Conference In Technical Disciplines, pp. 116-120, November 2013.

[17] R. G. Congalton, "A review of assessing the accuracy of classifications of remotely sensed data”, remote sens. environ., Vol. 37, pp. 35-46, 1991. 